\title{
HIV vaccines go to trial
}

\author{
Immune interventions capable of preventing or eradicating HIV infection have yet to achieve broad success in \\ humans. Renewed vigor in the clinical trial arena may bring us closer to that goal.
}

$\mathrm{n}$ n March, for only the second time to date, scientists reported long-term suppression of HIV-1 in the absence of antiretroviral therapy (ART) in an individual who had received a stem cell transplant from a donor with a homozygous mutation in the HIV-1 co-receptor C-C chemokine receptor type 5 (CCR5) (R. K. Gupta et al. Nature 568, 244-248, 2019). But transplantation of CCR5-defective cells, whether engineered or obtained from a stem cell donor, is not a viable prophylactic or therapeutic option for reasons that include safety, practicality and cost. We still need more widely applicable strategies that prevent new HIV-1 infections to curtail this pandemic.

Making pre-exposure prophylaxis (PrEP) more broadly available (59\% of all PrEP users are in the United States; https://prepwatch.org) and developing a preventive vaccine are two key clinical interventions that could interrupt the HIV transmission cycle. But while gains continue to be made in terms of early initiation of ART and development of long-acting and potently suppressive ART regimens to improve uptake and adherence, it is estimated that only $47 \%$ of $\mathrm{HIV}$-infected individuals are virally suppressed (www. unaids.org/en/resources/fact-sheet). This number highlights the need for alternative approaches to prevent viral transmission, yet a successful HIV vaccine has been elusive. Now, however, the landscape of HIV vaccines (and immunotherapies against the virus) may be poised to change.

The wide selection of preventive and therapeutic vaccines and therapies that are in or are entering phase 1 clinical trials was highlighted at the 2019 Joint Keystone Symposia on HIV and in recent reviews (M. Caskey, F. Klein \& M. C. Nussenzweig Nat. Med. 25, 547-553, 2019; G. Alter \& D. Barouch Cell Host Microbe 24, 25-33, 2018). Many of these studies will generate initial results over the next few years that may lay the ground work for a broader array of efficacy trials than exist to date (thus far, only six HIV vaccine efficacy trials have been completed). And given the lack of clinical success of HIV vaccines thus far, the enthusiasm in the field for advancing preclinical studies into clinical trials has seemed restrained. So the change in mood is very welcome and comes as a large number of groups are concurrently putting their vaccine concepts to the test.

Many viruses, including Adenovirus 4 (Ad4), Ad26, cytomegalovirus, and modified vaccinia virus Ankara, are slated for new clinical trials as vaccine vectors that deliver HIV-1 genes. These vectors will be used alone or, more commonly, in conjunction with the trimeric form of the HIV-1 envelope (Env) glycoprotein in order to elicit a balanced $\mathrm{T}$ cell and antibody response that many believe is required to block HIV from entering a cell. Another strategy moving into early-stage clinical trials involves testing sequential immunization with different variants of HIV-1 proteins intended to harness and drive maturation of germline antibodies through to the generation of broadly neutralizing antibodies (bNAbs). Meanwhile, other trials may involve alternative adjuvants or novel formulations using nanoparticles and liposomes, as well as intranasal immunization to boost mucosal immunity, and more conventional intramuscular delivery.

As the field transitions from animal models to human testing of this wealth of concepts, what can we expect to learn? Ideally, the vaccine studies will resolve whether, and at what magnitude, HIVspecific bNAbs can be elicited in humans by existing vaccine concepts, and the minimum vaccine requirements for their induction. They will also shed light on whether an approach that harnesses both $\mathrm{T}$ and $\mathrm{B}$ cell immunity gives rise to more potent and broad immune responses than a B cellfocused strategy.

But the phase 1 space is not limited to prophylactic strategies. Coupled to these efforts are ongoing or soon-to-launch trials of therapeutic approaches designed to eradicate existing HIV infection. These efforts include intravenous or subcutaneous delivery of HIV-specific bNAbs in infected adults or in HIV-exposed infants, infusion of novel bispecific antibodies that target both HIV and a human T cell antigen, and vaccination combined with a TLR7 agonist. Many of these trials are designed to include an ART interruption phase in which the effects of the intervention on the viral reservoir can be assessed.

These studies of therapeutic approaches will provide insight into whether and how rapidly the virus can evade immune pressure (exerted by bNAbs or by vaccination). They can also explore if the size and integrity of the viral reservoir is altered by the intervention, and the capacity of the immune system to restore control of virus replication in the absence of antiretroviral drugs. Clinical trials of passively transferred bNAbs in humans will provide much-needed evidence as to whether these antibodies alone are sufficient to prevent HIV acquisition or control existing infection.

Even with the encouraging plethora of new clinical trials, some hurdles remain. The challenge of overcoming HIV sequence diversity and immune escape is formidable; strategies targeting a single HIV-1 protein-as in some of the new trialsmay not be sufficient to confer protection against infection. Likewise, we do not yet fully understand the optimal $\mathrm{CD} 4^{+} \mathrm{T}$ cell response against HIV and the localization of this response that will be necessary to control-but not fuel by providing more targets-HIV infection. Ultimately, only efficacy studies will determine whether any of these promising concepts are capable of reducing infection rates or the levels of preexisting infection. But the pipeline of earlyphase clinical studies and accelerated pace of translation to the clinic are very encouraging developments for the field. Regardless of the outcomes of this first wave of trials, an acceleration of early-stage concepts to the clinic must be maintained for the successful treatment and prevention of HIV.

Published online: 8 May 2019 https://doi.org/10.1038/s41591-019-0460-0 